\title{
Optimization of Gain, Impedance, and Bandwidth of Yagi-Uda Array Using Particle Swarm Optimization
}

\author{
Munish Rattan, ${ }^{1}$ Manjeet Singh Patterh, ${ }^{2}$ and B. S. Sohi ${ }^{3}$ \\ ${ }^{1}$ Department of Electronics and Communication Engineering, Guru Nanak Dev Engineering College, Ludhiana, Punjab 141006, India \\ ${ }^{2}$ Department of Electronics and Communication Engineering, UCoE, Punjabi University, Patiala, Punjab 147002, India \\ ${ }^{3}$ Department of Electronics and Communication Engineering, UIET, Panjab University, Chandigarh 160014, India
}

Correspondence should be addressed to Munish Rattan, rattanmunish@gndec.ac.in

Received 20 September 2007; Accepted 20 February 2008

Recommended by Stefano Selleri

\begin{abstract}
Particle swarm optimization (PSO) is a new, high-performance evolutionary technique, which has recently been used for optimization problems in antennas and electromagnetics. It is a global optimization technique-like genetic algorithm (GA) but has less computational cost compared to GA. In this paper, PSO has been used to optimize the gain, impedance, and bandwidth of Yagi-Uda array. To evaluate the performance of designs, a method of moments code NEC2 has been used. The results are comparable to those obtained using GA.
\end{abstract}

Copyright ( $\odot 2008$ Munish Rattan et al. This is an open access article distributed under the Creative Commons Attribution License, which permits unrestricted use, distribution, and reproduction in any medium, provided the original work is properly cited.

\section{INTRODUCTION}

Yagi-Uda arrays are quite common in practice because they are light weight, simple to build, low cost, and provide moderately desirable characteristics for many applications. Yagi-Uda antenna was developed by Uda and Yagi during 1930s. Since then, it has received much attention in the literature. There are several gradient-based methods for optimization of Yagi antenna [1-3]. The shortcoming of gradient-based methods is that they are vulnerable to stuck in local optima. Also, these heavily depend on the choice of initial point, which depends on the experience of antenna designer. The solution to the problems of gradient-based methods can be overcome by using global optimization methods like genetic algorithm (GA) and particle swarm optimization (PSO). GA optimizers are particularly effective when the goal is to find an approximate global maximum in high dimension, multimodal function domain in near optimal manner. Haupt has compared GA and gradient-based methods for electromagnetic problems and found that the genetic algorithms are better than gradient-based methods [4]. Many reserachers have used GA for optimization of YagiUda array for different design objectives [5-9]. In [7], the Yagi-Uda array has been optimized for gain, impedance, and bandwidth using GA. In this paper, we are also achieving the same design objective using PSO.
PSO has been found to work better than GA in certain kind of optimization problems. Compared to GA, it is easily implemented and has least complexity [10]. Recently, it has been used for the synthesis of linear arrays [10, 11]. It has also been used for the optimization of gain, impedance, and relative side lobe level of Yagi-Uda array and has shown better performance than GA [12]. Bandwidth is also an important design objective, which needs to be explored along with gain and impedance. In this paper, PSO has been used for optimizing the gain, impedance, and bandwidth using length and spacing between elements as variables. Section 2 describes the PSO algorithm and the Yagi-Uda design optimization using PSO with simulation example. The work has been concluded in Section 4 .

\section{PARTICLE SWARM OPTIMIZATION}

PSO is an evolutionary algorithm based on the intelligence and cooperation of group of birds or fish schooling. It maintains a swarm of particles, where each particle represents a potential solution. In PSO, algorithm particles are flown through a multidimensional search space, where the position of each particle is adjusted according to its own experience and that of its neighbors. Table 1 shows some key terms used to describe PSO [13]. More details of algorithm can be found in $[14,15]$. 
TABLE 1: Key PSO vocabulary.

\begin{tabular}{|c|c|}
\hline \multicolumn{2}{|c|}{ Some key terms used to describe PSO } \\
\hline Particle/agent & $\begin{array}{l}\text { One single individual in the } \\
\text { swarm }\end{array}$ \\
\hline Location/position & $\begin{array}{l}\text { An agent's N-dimensional coor- } \\
\text { dinates which represents a solu- } \\
\text { tion to the problem }\end{array}$ \\
\hline Swarm & The entire collection of agents \\
\hline Fitness or cost & $\begin{array}{l}\text { A single number representing the } \\
\text { goodness of a given solution }\end{array}$ \\
\hline Personal best (pbest) & $\begin{array}{l}\text { The location in parameter space } \\
\text { of the best fitness returned for a } \\
\text { specific agent }\end{array}$ \\
\hline Global best (gbest) & $\begin{array}{l}\text { The location in parameter space } \\
\text { of the best fitness returned for the } \\
\text { entire swarm }\end{array}$ \\
\hline$V_{\max }$ & $\begin{array}{l}\text { The maximum allowed velocity } \\
\text { in a given direction }\end{array}$ \\
\hline
\end{tabular}

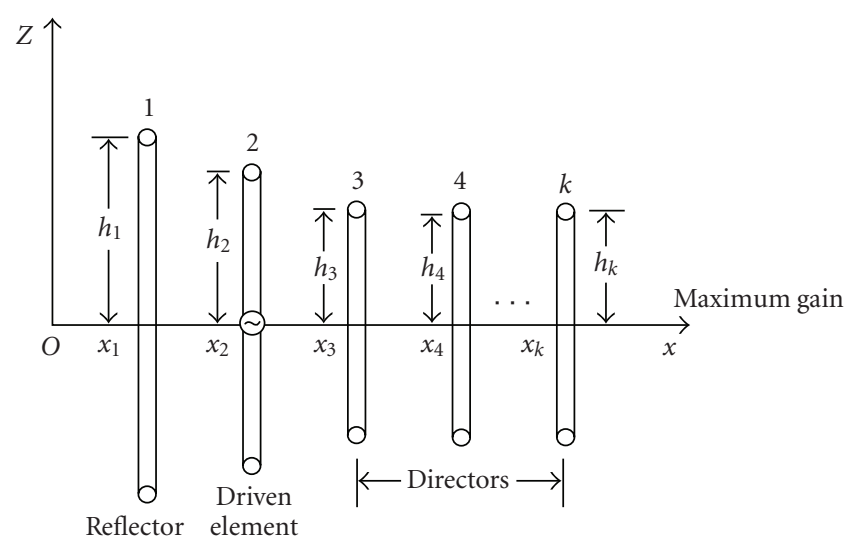

FIGURE 1: Geometry of a K element Yagi-Uda array.

\section{THE DESIGN PROCESS}

The goal of the design process is to develop an antenna that meets or exceeds some desired performance characteristics. A few of the characteristics that define the antenna performance are sidelobe level, beamwidth, bandwidth, front-toback ratio, size, gain, and input impedance. The quality of a design is expressed mathematically by an objective function. This paper uses the function which tries to maximize gain and to obtain optimum impedance, that is, $Z=50 \Omega$ for the $10 \%$ bandwidth. The objective function used is given by

$$
F=a G(x)-b|50-\operatorname{Re} Z(x)|-c|\operatorname{Im} Z(x)|,
$$

where $G$ is the Gain of antenna $x$ in the endfire direction; $Z$ is the impedance; and $|\cdot|$ denotes the absolute value. The value of $a$ was taken as 40 and $b$ and $c$ equal to 2. The values of $a$, $b$, and $c$ have been taken by hit and trail. It is clear that if the real part of impedance is far from 50 or the imaginary part is large, the fitness will be worse. To optimize the pattern for

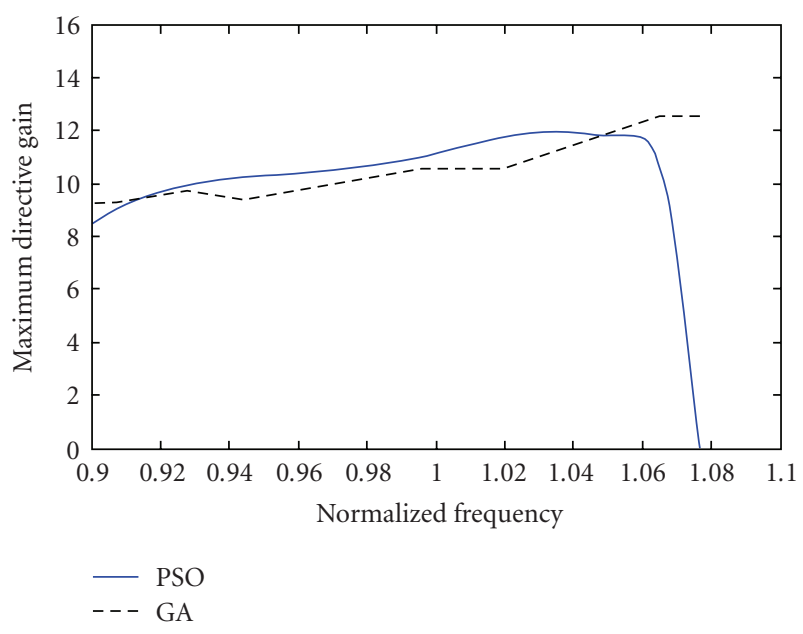

FIgURE 2: Maximum directive gain in $\mathrm{dB}$ versus normalized frequency for GA and PSO.

bandwidth, fitness operation is done in three frequencies: up, central, and down as in [7]. For example, if it is desired 10\% of bandwidth, the operation is made in three frequencies: $f_{\text {up }}$, $f_{c}$, and $f_{\text {down }}$, where, $f_{\text {up }}=1.05 * f_{c}$ and $f_{\text {down }}=.95 * f_{c}$.

Here, $f_{c}$, is the central frequency. Figure 1 shows the geometry of a $K$ element Yagi-Uda array. The six-element Yagi-Uda array has been optimized for gain, impedance, and bandwidth using PSO algorithm. The distances between consecutive elements (five different distances) and the lengths of each element are the parameters to be optimized. The problem with the antenna design is that if we increase gain, the impedance gets bad or bandwidth decreases. So, in order to have good antenna it should have high gain, low VSWR, and good bandwidth. The cross section radius is the same for all elements and is set equal to $0.003369 \lambda$ wavelengths at $299.8 \mathrm{MHz}$. The NEC2 simulation program [6] has been used to evaluate all antenna designs. The source element for excitation was specified to be the middle segment of the driven element. The $z$ location of the reflector element was always set to 0 . The antenna was analyzed in free space. The spacing between elements was allowed to vary between $0.10 \lambda$ and $0.45 \lambda$, and the length of each element is allowed to vary between $0.15 \lambda$ and $0.35 \lambda$. Figure 2 shows the maximum directive gain versus frequency response for GA and PSO. As can be seen, PSO shows better gain characteristics compared to GA. Figure 3 shows the VSWR versus frequency for GA and PSO. It can be observed that GA has better impedance characteristics. But it has to be noted that in [7] the position of feedpoint has been varied for getting better impedance characteristics, whereas in this work the feedpoint position is fixed. Table 2 shows the relative performance comparisons. The convergence rate of the PSO has been shown in Figure 4. In general, PSO converges in a few iterations compared to GA since it has less complexity and can be easily implemented. In this very particular problem, PSO converges after 2500 iterations, whereas GA took 10000 iterations. This results in considerable time saving. The memory complexity is also less in PSO as PSO is inherently less complex compared to GA. 
TABLE 2: Length, spacing, and performance comparisons. The central frequency is $300 \mathrm{mHz}$. The radius of elements is $0.003369 \lambda$. Length and spacing are also in terms of wavelength.

\begin{tabular}{lcccc}
\hline & \multicolumn{2}{c}{ GA optimized gain, impedance, and bandwidth [7] } & \multicolumn{2}{c}{ PSO optimized for gain, impedance, and bandwidth } \\
\hline Element & Length & Spacing & Length & Spacing \\
1 & .52 & - & .4939 & - \\
2 & .47 & .182 & .4665 & .2269 \\
3 & .42 & .152 & .4213 & .1662 \\
4 & .41 & .229 & .4149 & .2883 \\
5 & .39 & .435 & .4120 & .3213 \\
6 & .39 & .272 & .4199 & .2731 \\
Feedpoint & & & 10.22 & 0 \\
Gain $(\mathrm{dBi})\left(f_{\text {down }} / f_{\text {up }}\right)$ & 9.39 & 11.29 & $49.08-j 20$ & 12.12 \\
$Z(\Omega)$ & $52.4-j 6.6$ & $47.1+j 2.6$ & $49.99+j 0.50$ \\
\hline
\end{tabular}

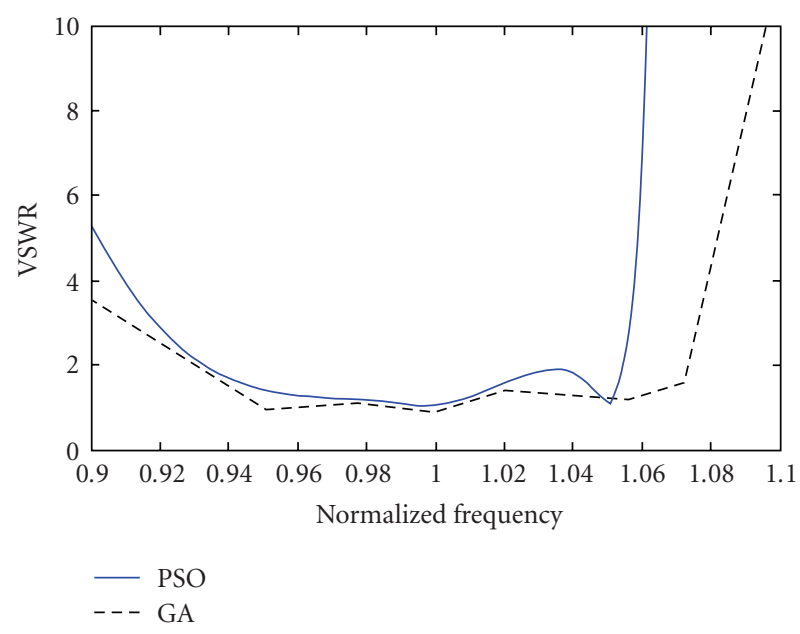

FIGURE 3: Voltage standing wave ratio versus normalized frequency for GA and PSO.

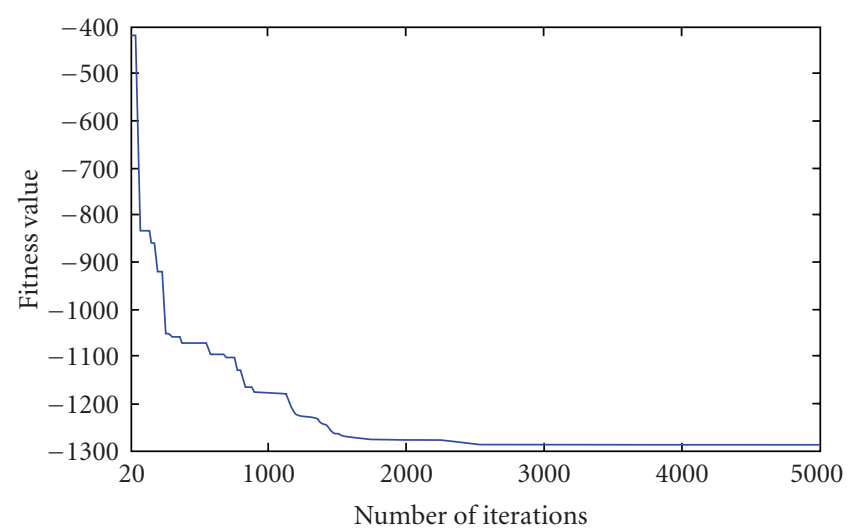

FIgURE 4: Convergence rate of the PSO algorithm, showing fitness value versus number of iterations.

\section{CONCLUSION}

Particle swarm optimization is currently being used for antenna optimization due to its low complexity and global nature. Earlier designs using PSO did not consider bandwidth as optimization variable. The bandwidth is an important parameter as antennas work in the specific frequency bands. In the present work, PSO has been successfully used for the optimization of gain, impedance, and bandwidth of Yagi-Uda array. Results show that PSO is well suited for these kinds of multimodal problems. The results are comparable to those obtained using GA taking length and spacing as variable. More control over the pattern can be obtained by taking length, spacing, and radii, that is, all three as variables. Also, other design objectives like sidelobe level and beamwidth can be included in the optimization process.

\section{REFERENCES}

[1] D. K. Cheng and C. A. Chen, "Optimum element spacings for Yagi-Uda arrays," IEEE Transactions on Antennas and Propagation, vol. 21, no. 5, pp. 615-623, 1973.

[2] C. A. Chen and D. K. Cheng, "Optimum element lengths for Yagi-Uda arrays," IEEE Transactions on Antennas and Propagation, vol. 23, no. 1, pp. 8-15, 1975.

[3] D. K. Cheng, "Gain optimization for Yagi-Uda arrays," IEEE Antennas and Propagation Magazine, vol. 33, no. 3, pp. 42-46, 1991.

[4] R. L. Haupt, "Comparison between genetic and gradientbased optimization algorithms for solving electromagnetics problems," IEEE Transactions on Magnetics, vol. 31, no. 3, pp. 1932-1935, 1995.

[5] E. A. Jones and W. T. Joines, "Design of Yagi-Uda antennas using genetic algorithms," IEEE Transactions on Antennas and Propagation, vol. 45, no. 9, pp. 1386-1392, 1997.

[6] G. J. Burke and A. J. Poggio, "Numerical electromagnetics code (NEC) - method of moments," Tech. Rep. UCID18834, Lawrence Livermore National Laboratory, Livermore, Calif, USA, 1981.

[7] D. Correia, A. J. M. Soares, and M. A. B. Terada, "Optimization of gain, impedance and bandwidth in Yagi-Uda antennas using genetic algorithm," in Proceedings of the International Microwave and Optoelectronics Conference (IMOC '99), vol. 1, pp. 41-44, Rio de Janeiro, Brazil, August 1999.

[8] J. D. Lohn, W. F. Kraus, D. S. Linden, and S. P. Colombano, "Evolutionary optimization of Yagi-Uda antennas," in Proceedings of the 4th International Conference on Evolvable Systems (ICES '01), pp. 236-243, Tokyo, Japan, October 2001. 
[9] Y. Kuwahara, "Multiobjective optimization design of Yagi-Uda antenna," IEEE Transactions on Antennas and Propagation, vol. 53, no. 6, pp. 1984-1992, 2005.

[10] M. M. Khodier and C. G. Christodoulou, "Linear array geometry synthesis with minimum sidelobe level and null control using particle swarm optimization," IEEE Transactions on Antennas and Propagation, vol. 53, no. 8, pp. 2674-2679, 2005.

[11] M. H. Bataineh and J. I. Ababneh, "Synthesis of aperiodic linear phased antenna arrays using particle swarm optimization," Electromagnetics, vol. 26, no. 7, pp. 531-541, 2006.

[12] S. Baskar, A. Alphones, P. M. Suganthan, and J. J. Liang, "Design of Yagi-Uda antennas using comprehensive learning particle swarm optimisation," IEE Proceedings: Microwaves, Antennas and Propagation, vol. 152, no. 5, pp. 340-346, 2005.

[13] J. Robinson and Y. Rahmat-Samii, "Particle swarm optimization in electromagnetics," IEEE Transactions on Antennas and Propagation, vol. 52, no. 2, pp. 397-407, 2004.

[14] N. P. Padhy, Artificial Intelligence and Intelligent Systems, chapter 10, Oxford University Press, New York, NY, USA, 2nd edition, 2005.

[15] J. Kennedy and R. C. Eberhart, "Particle swarm optimization," in Proceedings of the IEEE International Conference on Neural Networks (ICNN '95), vol. 4, pp. 1942-1948, Perth, Western Australia, November 1995. 

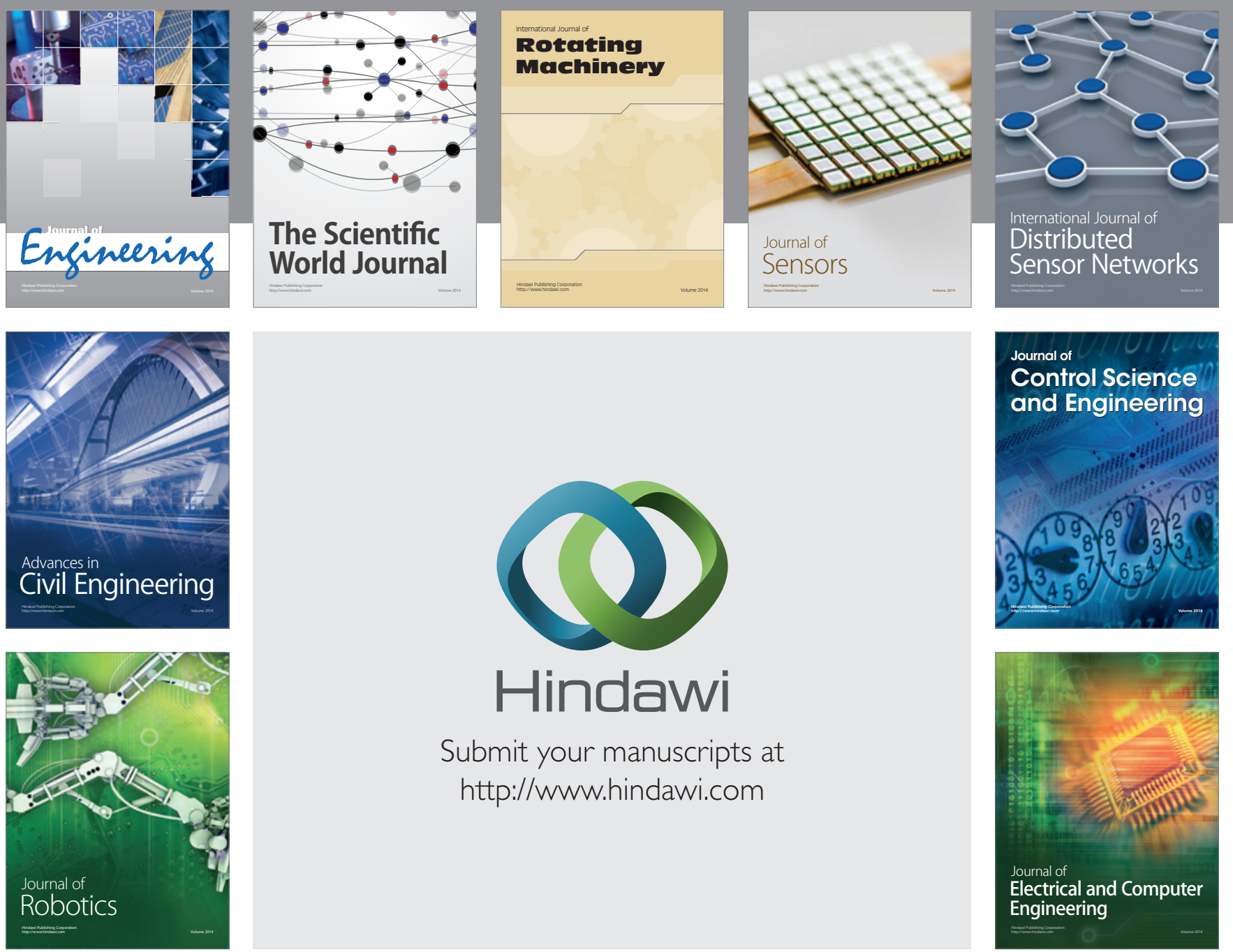

Submit your manuscripts at

http://www.hindawi.com
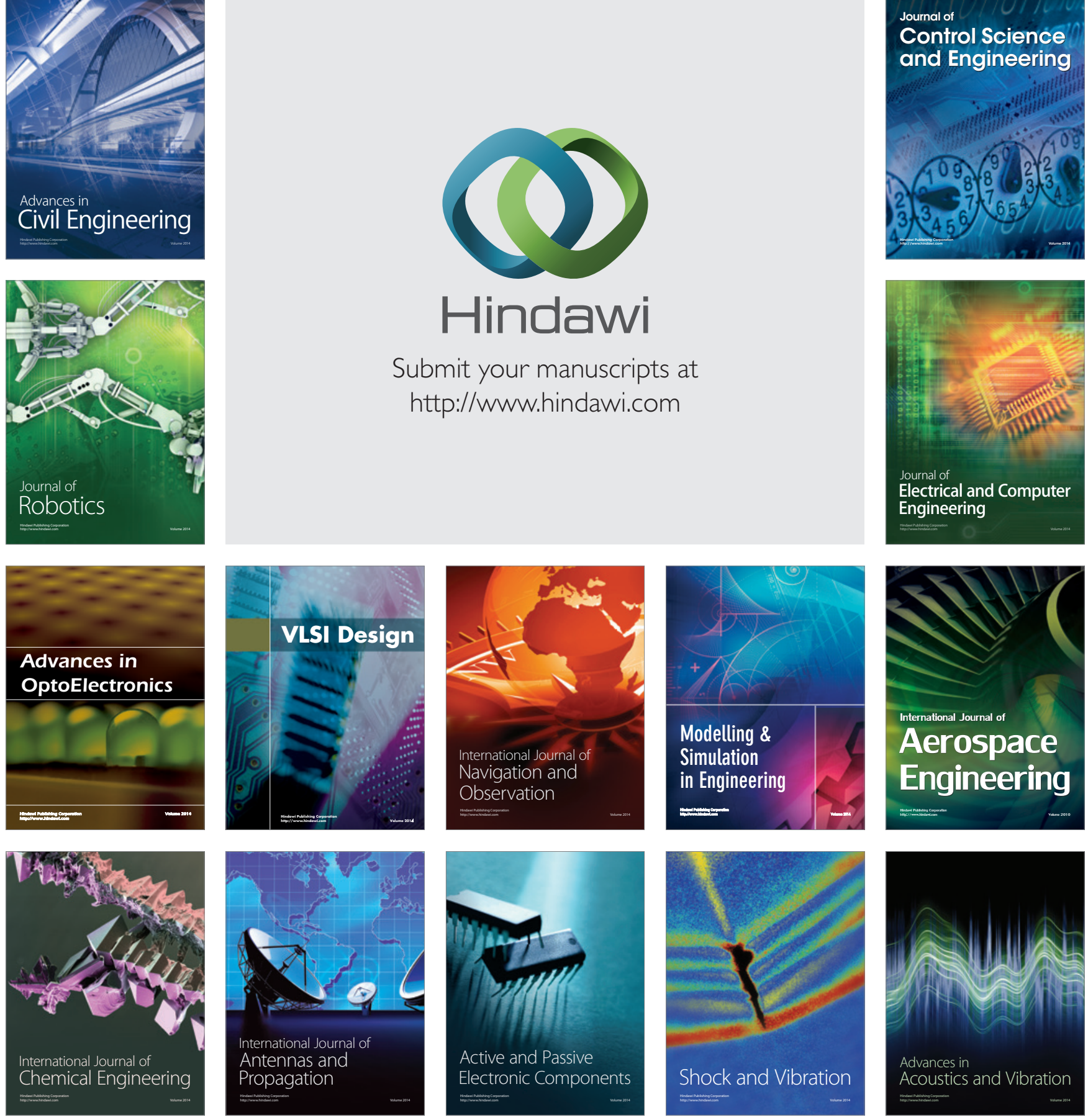